\title{
In vivo measurement of apolipoprotein E from the brain interstitial fluid using microdialysis
}

\author{
Jason D Ulrich 1,2,4, Jack M Burchett ${ }^{1,2,4}$, Jessica L Restivo ${ }^{1,2,4}$, Dorothy R Schuler ${ }^{1,2,4}$, Philip B Verghese ${ }^{1,2,4}$ \\ Thomas E Mahan ${ }^{1,2,4}$, Gary E Landreth ${ }^{5}$, Joseph M Castellano ${ }^{1,2,4}$, Hong Jiang ${ }^{1,2,4}$, John R Cirrito ${ }^{1,2,4}$ \\ and David M Holtzman ${ }^{1,2,3,4^{*}}$
}

\begin{abstract}
Background: The APOE4 allele variant is the strongest known genetic risk factor for developing late-onset Alzheimer's disease. The link between apolipoprotein $E$ (apoE) and Alzheimer's disease is likely due in large part to the impact of apoE on the metabolism of amyloid $\beta(A \beta)$ within the brain. Manipulation of apoE levels and lipidation within the brain has been proposed as a therapeutic target for the treatment of Alzheimer's disease. However, we know little about the dynamic regulation of apoE levels and lipidation within the central nervous system. We have developed an assay to measure apoE levels in the brain interstitial fluid of awake and freely moving mice using large molecular weight cut-off microdialysis probes.

Results: We were able to recover apoE using microdialysis from human cerebrospinal fluid (CSF) in vitro and mouse brain parenchyma in vivo. Microdialysis probes were inserted into the hippocampus of wild-type mice and interstitial fluid was collected for 36 hours. Levels of apoE within the microdialysis samples were determined by ELISA. The levels of apoE were found to be relatively stable over 36 hours. No apoE was detected in microdialysis samples from apoE KO mice. Administration of the RXR agonist bexarotene increased ISF apoE levels while ISF A $\beta$ levels were decreased. Extrapolation to zero-flow analysis allowed us to determine the absolute recoverable concentration of apoE3 in the brain ISF of apoE3 KI mice. Furthermore, analysis of microdialysis samples by non-denaturing gel electrophoresis determined lipidated apoE particles in microdialysis samples were consistent in size with apoE particles from CSF. Finally, we found that the concentration of apoE in the brain ISF was dependent upon apoE isoform in human apoE KI mice, following the pattern apoE2>apoE3>apoE4.
\end{abstract}

Conclusions: We are able to collect lipidated apoE from the brain of awake and freely moving mice and monitor apoE levels over the course of several hours from a single mouse. Our technique enables assessment of brain apoE dynamics under physiological and pathophysiological conditions and in response to therapeutic interventions designed to affect apoE levels and lipidation within the brain.

Keywords: Microdialysis, Apolipoprotein E, Alzheimer's disease

\section{Background}

Intercellular lipid transport in the central nervous system (CNS) is predominantly mediated by high-density lipoprotein (HDL)-like particles containing the lipidbinding protein apolipoprotein $\mathrm{E}$ (apoE) [1]. Within the CNS, apoE is secreted primarily by astrocytes [2]. In humans there are three common $A P O E$ allele variants:

\footnotetext{
*Correspondence: holtzman@neuro.wustl.edu

'Department of Neurology, Saint Louis, MO, USA

${ }^{2}$ Hope Center for Neurological Disorders, Saint Louis, MO, USA

Full list of author information is available at the end of the article
}

APOE2, APOE3, and APOE4. Possession of the $\varepsilon 4$ allele is the strongest known genetic risk factor for developing late-onset Alzheimer's disease (LOAD), while apoE2 is protective[2]. ApoE likely influences $\mathrm{AD}$ in large part through the isoform-dependent effects of apoE on the metabolism of amyloid- $\beta$ (A $\beta)$ in the brain. ApoE isoforms differentially influence the rate of $A \beta$ clearance from the brain with the order of clearance being apoE4<apoE3<apoE2 [3]. ApoE also facilitates the formation of $A \beta$ aggregates (e.g. oligomers and fibrils) within the brain, which are thought to induce synaptic

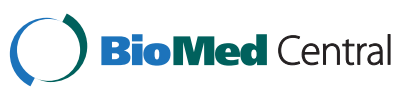


and neuronal toxicity [4]. In addition to isoformdependent effects, the degree of apoE lipidation and apoE concentration within the brain also influences $A \beta$ plaque deposition and $A \beta$ clearance [5-8]. ApoE likely plays additional roles in the brain in processes such as synaptic plasticity, regulation of extracellular lipid metabolism, and inflammation (for review, see [2]).

Despite the importance of apoE in $\mathrm{AD}$, little is known about the physiological regulation of apoE within the CNS. To date, analysis of the regulation of apoE expression and lipidation within the CNS has been limited to in vitro cell culture systems and assessment of apoE from homogenized brain extracts or cerebral spinal fluid (CSF). Thus, very little is known about the dynamic regulation of apoE within the brain, particularly within the interstitial fluid (ISF), where apoE is present immediately after secretion from astrocytes and where it likely functionally interacts with $\mathrm{A} \beta$ and other molecules. Given recent interest in apoE as a therapeutic target for $\mathrm{AD}$, there is a critical need to develop methods to dynamically assess the levels, lipidation, and effects of apoE within the brain in vivo $[8,9]$.

Microdialysis is a widely used and powerful technique that has been employed to study neurotransmitters and other small molecules or peptides within the brain [10]. In $\mathrm{AD}$, microdialysis has been instrumental in studying the dynamics of $\mathrm{A} \beta$ production and clearance in vivo [3,11-14]. However, the comparatively large size of lipidated apoE particles $(\sim 300 \mathrm{kDa})$ has impeded the utility of microdialysis to similarly assess apoE metabolism within the brain. A recent report demonstrated that a high molecular weight cut-off (MWCO) microdialysis probe was capable of collecting small $(<30 \mathrm{kDa})$ proteins from the brain ISF[15]. We hypothesized that such a probe might be useful for collecting lipidated lipoproteins. Here, we report the use of the high MWCO AtmosLM microdialysis probe to collect and quantify lipidated apoE particles from the brain ISF of awake and freely moving animals by in vivo microdialysis. Non-denaturing gel electrophoresis analysis of the ISF samples indicates that apoE-containing particles are heterogeneous in size and are consistent in size with apoE-containing particles from CSF. Using this technique, we found that the level of apoE in the hippocampal ISF is dependent upon apoE isoform. The ability to dynamically measure apoE levels and lipidation in the brain ISF will be useful for studying lipid metabolism within the brain ISF and the molecular basis for the effect of apoE on AD pathology.

\section{Results and discussion}

The relatively large hydrated diameter of lipidated apoE particles prohibits collection by commonly used lower MWCO microdialysis probes (typically 6-40 kDa). To circumvent this issue, we optimized the use of a 1,000 $\mathrm{kDa}$ MWCO microdialysis probe for collection of apoE. We first characterized the efficiency of apoE collection from human CSF using microdialysis in vitro. The recovery of analyte by microdialysis is inversely related to the flow rate through the microdialysis probe[10]. By extrapolating along the recovery curve to a zero flow rate, it is possible to estimate the maximum amount of exchangeable analyte [10]. Microdialysis samples were collected hourly at flow rates ranging from $0.4 \mu \mathrm{L} / \mathrm{min}$ to $1.6 \mu \mathrm{L} / \mathrm{min}$. As expected, decreasing the flow rate increased the amount of apoE detected in microdialysis samples (Figure 1A). We estimated the total amount of exchangeable apoE to be $111.2 \pm 14.4 \mathrm{ng} / \mathrm{mL}$, which represented $\sim 2 \%$ of the $6024 \pm 152.3 \mathrm{ng} / \mathrm{mL}$ total apoE detected within the CSF samples.

Having determined it was possible to collect apoE by microdialysis using a $1,000 \mathrm{kDa}$ MWCO probe, we next tested whether we could collect apoE in vivo. We implanted microdialysis probes into the hippocampus of 34 month old wild-type mice and collected microdialysis samples every two hours at a constant flow rate of $1.0 \mu \mathrm{L} /$ min. Under these conditions, levels of apoE within the hippocampal ISF were stable throughout the 36 hour collection duration (Figure 1B). Similar to previous studies, we observed only minimal astrogliosis in the cortex proximal to the site of cannula insertion and no evidence of substantial inflammation following microdialysis (data not shown) $[14,16]$. We found the mean concentration of recoverable apoE in the hippocampal ISF to be $10.1 \pm 1.8$ $\mathrm{ng} / \mathrm{mL}$ at a constant flow rate of $1.0 \mu \mathrm{L} / \mathrm{min}$ (Figure $1 \mathrm{C}$ ). To verify the specificity of our assay we also performed microdialysis in apoE $\mathrm{KO}$ mice. As expected, we detected no apoE in the apoE KO mouse ISF dialysate (Figure $1 \mathrm{C}$ ). These data confirm that we are able to collect apoE from the brain ISF and specifically measure how it changes over the course of several hours in a single mouse.

Due to the relatively high variance we observed in apoE levels, we performed a power analysis to determine if we could feasibly detect statistically significant differences in ISF apoE levels. Given the observed mean concentration and average standard deviation of murine ISF apoE at a given time point, we estimate $\sim 5-6$ animals per group would be required to observe a statistically significant $70 \%$ change in apoE levels at an $\alpha$-value of 0.80 and p-value of 0.05 . Furthermore, because in vivo microdialysis enables assessment of changes in apoE levels in a single mouse, normalizing the level of apoE detected to a baseline value (i.e. the mean level from first 9 hours of collection) for each animal eliminates inter-animal variability in apoE levels and allows for detection of a statistically significant $50 \%$ change in relative apoE levels with $\sim 5-6$ animals.

Recent studies propose that modulating apoE levels and lipidation in the brain using nuclear hormone 

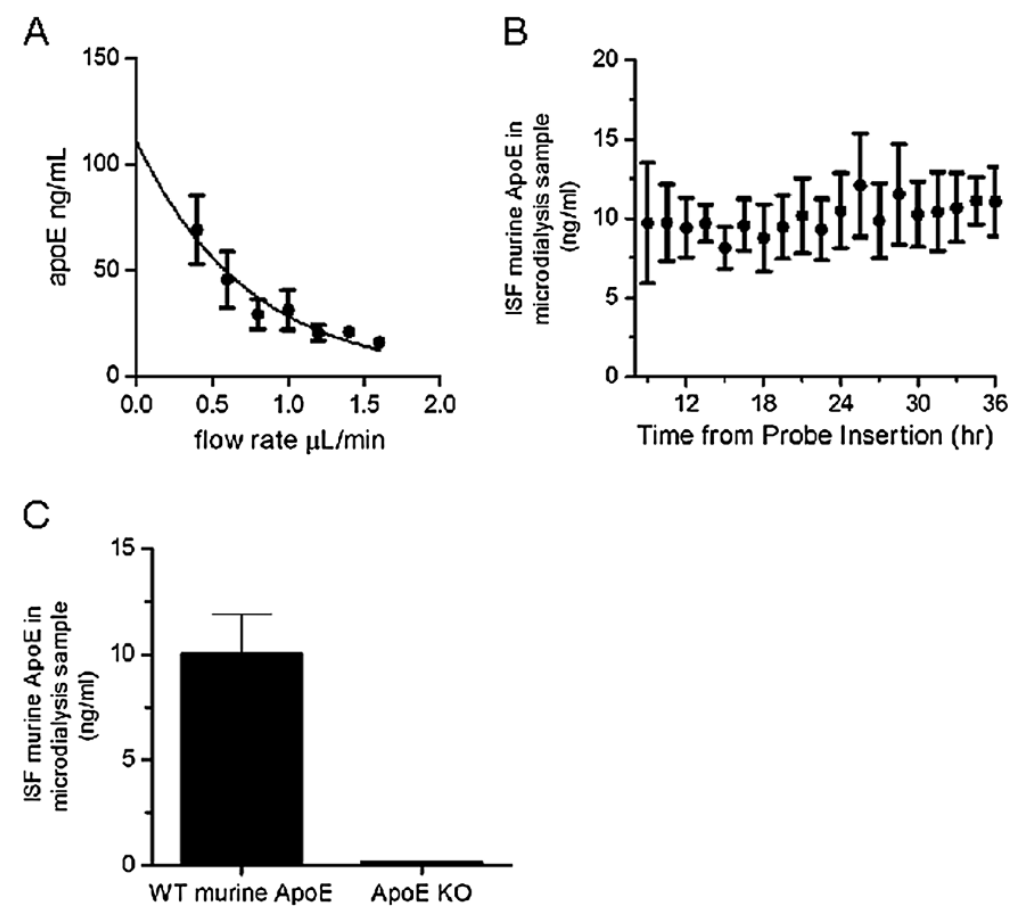

Figure 1 Analysis of apoE levels by microdialysis in vitro and in vivo. A. Microdialysis samples were collected hourly from human CSF in vitro at flow rates ranging from $0.4 \mu \mathrm{L} / \mathrm{min}$ to $1.6 \mu \mathrm{L} / \mathrm{min}$. The concentration of apoE within microdialysis samples and CSF was determined by ELISA. Data points represent mean \pm SEM $(n=3)$. A single-phase exponential decay curve $\left(r^{2}=0.93\right)$ was used to calculate the estimated mean concentration of apoE at zero flow. B. Microdialysis probes were implanted into the hippocampus of 3-4 month old mice and ISF collected bihourly for 36 hours. The concentration of apoE within microdialysis samples was determined by ELISA. Data points represent mean \pm SEM ( $n=5$ ). C. The mean concentration of murine apoE in ISF microdialysis samples from WT ( $n=5)$ and apoE KO mice $(n=2)$ was determined by ELISA. ISF samples were collected at a constant flow rate of $1.0 \mu \mathrm{L} / \mathrm{min}$. Data are presented as mean $\pm \mathrm{SEM}$.

receptor agonists may be an effective therapeutic target for $\mathrm{AD}[8,17]$. We tested whether we could detect changes in ISF apoE and A $\beta$ levels following administration of the retinoid-X-receptor (RXR) agonist bexarotene using microdialysis. We monitored hippocampal ISF apoE and $\mathrm{A} \beta$ levels in 2-month old APP/PS1 mice.
Following establishment of a 6-hour baseline apoE and $\mathrm{A} \beta$ level, bexarotene $(100 \mathrm{mg} / \mathrm{kg})$ or vehicle (water) were administered to the mice via oral gavage. Bexarotene treatment led to a steady increase in ISF apoE levels beginning $~ 12$ hours post-administration (Figure 2A). ISF apoE levels were increased 2.5-fold 30-36 hours post-
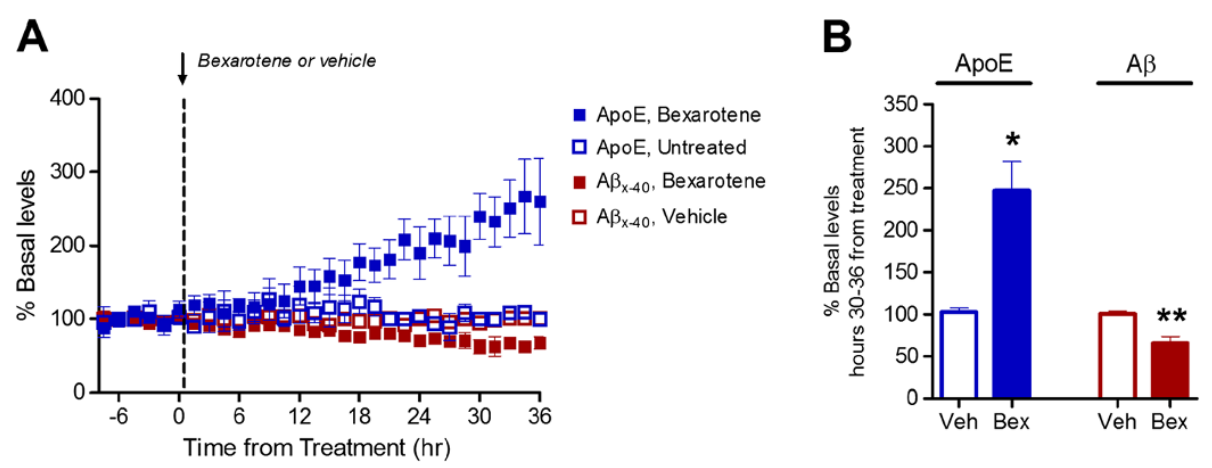

Figure 2 Bexarotene increases ISF apoE levels and decreases ISF A $\beta$ levels. A. ISF A $\beta_{x-40}$ and apoE levels in the hippocampus of 2-month old APP/PS1 mice were monitored using in vivo microdialysis. Following establishment of a 6 hour baseline ISF level for $A \beta_{x-40}$ and apoE, mice were administered bexarotene (100 mg/kg p.o.) or vehicle (water) and ISF A $\beta_{x-40}$ and apoE levels assessed for an additional 36 hours. B. The mean percent change from baseline of ISF apoE and A $\beta_{x-40}$ levels 30-36 post-administration was compared between vehicle and bexarotene treated mice. Bexarotene significantly increased ISF apoE levels $(247 \pm 34.3 \%, n=2)$ compared to vehicle $(103 \pm 5.2 \%, n=3)\left({ }^{*} p<0.05\right.$, unpaired t-test). Bexarotene decreased ISF $A \beta$ levels $(65.1 \pm 8.1 \%, n=3)$ compared to vehicle $(100 \pm 3.7 \%, n=6)$. ${ }^{* *} p<0.005$, unpaired t-test). 
treatment (Figure 2A and B). ISF A $\beta$ levels were decreased by $\sim 35 \%$, similar to previous observations (Figure $2 \mathrm{~A}$ and B) [8]. These data demonstrate the utility of microdialysis to detect biologically relevant, pharmacologically-induced changes in ISF apoE levels.

To further validate our technique we tested whether we could determine an absolute concentration of recoverable apoE within the hippocampal ISF by the extrapolated zero-flow method. We implanted microdialysis probes into the hippocampus of 3-4 month old apoE3 KI mice and collected ISF dialysate at flow rates ranging from $0.4 \mu \mathrm{L} / \mathrm{min}$ to $1.6 \mu \mathrm{L} / \mathrm{min}$. We then fit a singleexponential decay curve to the concentration of apoE as a function of flow rate (Figure 3A). Our analysis determined the concentration of recoverable apoE3 in the ISF under steady-state conditions was $38.1 \pm 4.0 \mathrm{ng} / \mathrm{mL}(\mathrm{n}=4)$.

ApoE particles in the CSF or secreted by cultured astrocytes are a heterogeneous mixture that vary in size depending upon the degree of lipidation [5,18-20]. We compared the lipidation of apoE3 particles collected from brain parenchymal ISF to apoE3 particles in CSF by non-denaturing gel electrophoresis, which separates proteins based upon their hydrated diameter. As previously reported, apoE3 particles in CSF were heterogeneous in size, ranging from $8.1 \mathrm{~nm}$ to $17.0 \mathrm{~nm}$ (Figure $2 \mathrm{~B}$ ) [21]. ApoE3 particles collected from ISF were lipidated and were similar in size to particles found in CSF (Figure 3B). We further verified that the lipidated apoE3 particles in ISF samples were not due to contamination from the artificial cerebral spinal fluid (aCSF) used for microdialysis (Figure 3B).

Previous studies found an isoform-dependent effect on apoE levels in the hippocampus, cortex, and CSF in apoE KI mice with apoE2-expressing mice having the highest levels of apoE and apoE4 expressing mice the lowest $[22,23]$. Therefore, we tested whether we could detect isoform-dependent differences in apoE levels in the hippocampal ISF by microdialysis. We implanted microdialysis probes into the hippocampus of 3-4 month old apoE2, apoE3 or apoE4 KI mice and assessed the absolute concentration of human apoE in the ISF dialysate by ELISA. The concentration of apoE2 within the ISF $(46.7 \pm 15.6 \mathrm{ng} / \mathrm{mL}$, $\mathrm{n}=3)$ was significantly greater than that of apoE4 (12.6 \pm $1.8 \mathrm{ng} / \mathrm{mL}, \mathrm{n}=4)$ and the concentration of apoE3 $(18.7 \pm$ $5.3, n=6)$ was at a level between that of apoE2 and apoE4 (Figure 4A). To confirm that the observed differences were not due to differential detection of apoE isoforms by the antibodies used in the ELISA, we measured equal amounts of recombinant apoE2, apoE3, and apoE4 by ELISA and found no significant difference in detection among the apoE isoforms (Figure 4B). Therefore, these results support the utility of our assay for observing differences in apoE levels in the ISF that are similar to those seen in CSF and in the brain.

\section{Conclusions}

We have developed a sensitive microdialysis assay for assessing the levels and lipidation of apoE within the
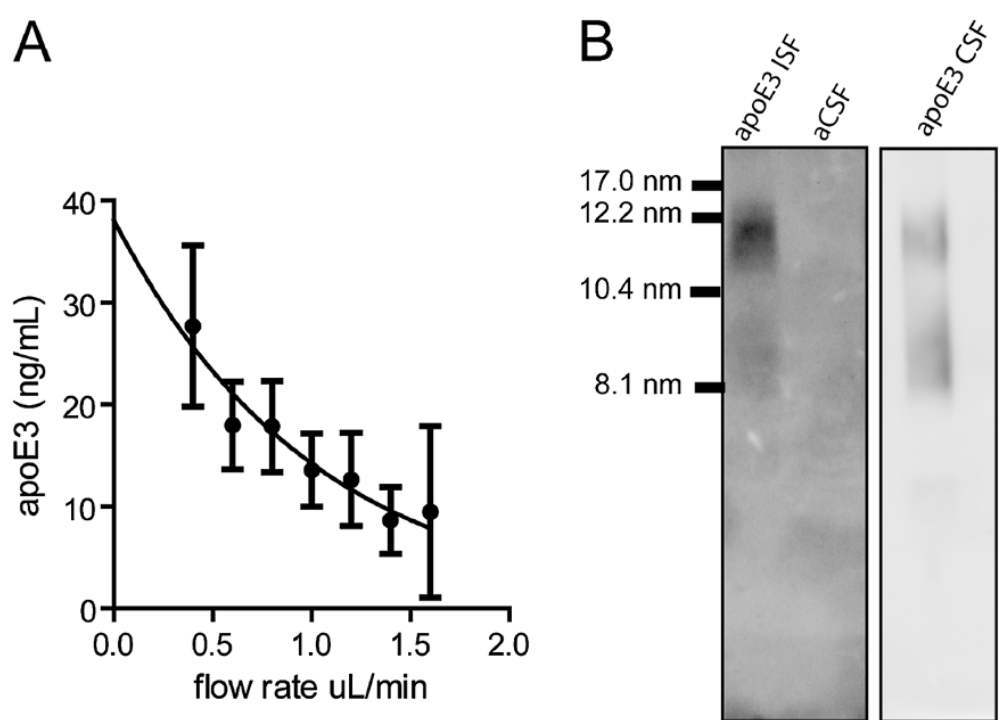

Figure 3 Analysis of hippocampal ISF apoE3 levels and lipidation. A. The apoE concentration in microdialysis samples collected at flow rates ranging from $0.4 \mu \mathrm{L} / \mathrm{min}$ to $1.6 \mu \mathrm{L} /$ min was determined by ELISA. Data are presented as mean \pm SEM $(\mathrm{n}=4)$. A single-phase exponential decay curve $\left(r^{2}=0.93\right)$ was used to calculate the estimated mean concentration of apoE3 at zero flow. B. The lipidation of apoE in ISF and CSF samples was analyzed by non-denaturing gel electrophoresis using 4-20\% Tris-glycine gradient gels. No apoE was detected in the aCSF used for microdialysis. The samples were run on the same gel; however the CSF panel is from a shorter exposure than the ISF panel for clarity. 

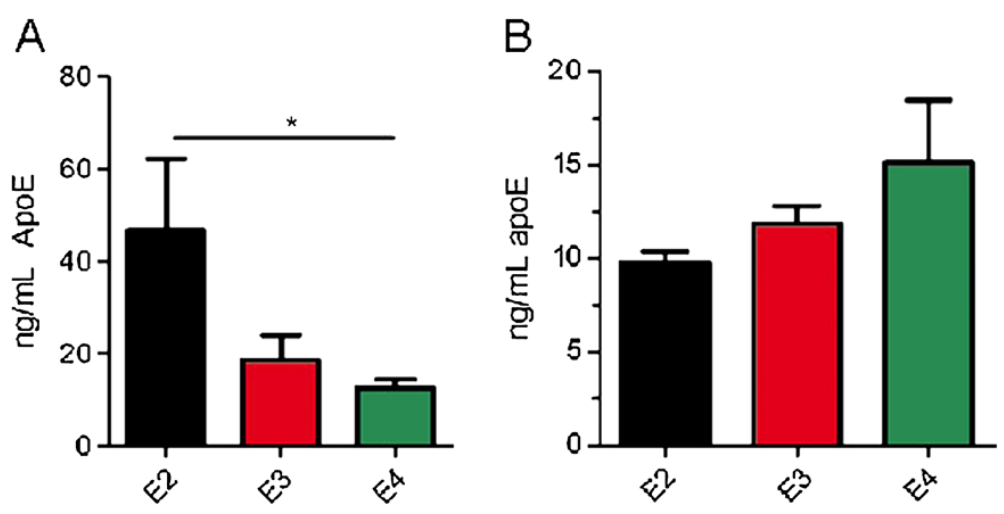

Figure 4 Isoform-dependent differences in apoE levels in the hippocampal ISF. A. ISF samples from the hippocampus of apoE2 KI, apoE3 $\mathrm{Kl}$, and apoE4 KI mice were obtained by microdialysis using a constant flow-rate of $1.0 \mu \mathrm{L} / \mathrm{min}$. ApoE levels were assessed by ELISA and compared by ANOVA followed by Tukey's post hoc test. ${ }^{*} \mathrm{p}<0.05, \mathrm{n}=3-6$ mice per genotype. Data are presented as mean $\pm \mathrm{SEM}$. B. $12.5 \mathrm{ng} / \mathrm{mL}$ of recombinant apoE2, apoE3, or apoE4 was measured by ELISA and compared by ANOVA ( $p=0.22$ ).

brain parenchymal ISF in awake and freely moving animals. Despite being the strongest genetic risk factor for developing late-onset $\mathrm{AD}$, the precise molecular mechanism by which $A P O E$ genotype influences the risk of developing $\mathrm{AD}$ remains unknown. Previous studies indicate that apoE affects the metabolism and oligomerization of $\mathrm{A} \beta$ within the brain $[3,4,24]$ as well as influences a variety of other biological processes [2]. Recent studies have therapeutically targeted the levels and lipidation of apoE to enhance clearance of $A \beta$ from the brain [8]. The method described here will be useful in sampling ISF apoE levels over time in order to assess the relationship between apoE levels and lipidation and $A \beta$ metabolism in the brain. Furthermore, assessment of apoE by microdialysis could prove useful in investigating the regulation of extracellular lipid homeostasis within the brain. This may be important in synaptic plasticity and repair after brain injury, as well as potentially play a role in neurodegenerative diseases such as Niemann-Pick disease [25].

\section{Methods}

\section{Materials}

Mouse apoE monoclonal antibodies $(\mathrm{mHJ}$ 6.1, $\mathrm{mHJ}$ 6.2, and $\mathrm{mHJ}$ 6.3) were generated in-house and have been described previously [9]. Polyclonal apoE antibody (50A-G1b) was purchased from Academy Bio-medical Co. (Houston, TX, USA). Recombinant apoE3 was purchased from Leinco Technologies (St. Louis, MO, USA). Bexarotene $\left(\right.$ Targretin $^{\mathrm{TM}}$ ) was provided by Dr. Gary Landreth (Case Western Reserve University).

\section{Animals}

ApoE2, apoE3, and apoE4 knock-in (KI) mice were generously provided by Dr. Patrick M. Sullivan (Duke University) and have been previously described [26]. To assess murine apoE, wild type mice on a mixed C57BL6/
$\mathrm{C} 3 \mathrm{H}$ background were utilized (Jackson Labs). APPswe/ PS1 $\delta$ E9 mice (APP/PS1) and ApoE knock-out (KO) mice were obtained from Jackson Labs. Mice were housed under constant light/dark conditions and had access to food and water ad libitum. All experimental protocols were approved by the Animal Studies Committee at Washington University in St. Louis.

\section{In vitro microdialysis}

Human CSF was collected by lumbar puncture from cognitively normal research volunteers from the Washington University Memory and Aging Project [27]. 1,000 kDa MWCO membrane microdialysis probes (AtmosLM Microdialysis probe, PEP-X-0Y, Eicom, San Diego, CA, USA) were connected to a two-channel peristaltic pushpull pump (MAB20, SciPro, Sanborn, NY, USA). The inlet-port of the microdialysis probe was connected to the push channel of the pump using Joint Teflon Tubing (inner diameter $0.1 \mathrm{~mm}$ ) (JT-10-100, Eicom, San Diego, CA, USA) and the outlet-port was connected to the pull channel of the pump using Teflon (FEP) tubing (inner diameter $0.12 \mathrm{~mm}$ ). The flow rates of the push and pull channels were calibrated to within 5\% tolerance. The probes were then flushed with microdialysis perfusion solution (4\% human albumin (Gemini Bioproducts, West Sacramento, CA, USA)-containing artificial CSF (aCSF) (in mM: $1.3 \mathrm{CaCl}_{2}, 1.2 \mathrm{MgSO}_{4}, 3 \mathrm{KCl}, 0.4 \mathrm{KH}_{2} \mathrm{PO}_{4}, 25$ $\mathrm{NaHCO}_{3}, 122 \mathrm{NaCl}, \mathrm{pH} 7.35$ ) that had been prepared on the day of use and filtered through a $0.1 \mu \mathrm{m}$ PES membrane. Microdialysis was performed at flow rates of $0.4 \mu \mathrm{L} / \mathrm{min}$ to $1.6 \mu \mathrm{L} / \mathrm{min}$ and samples were collected hourly using a refrigerated fraction collector (Univentor 820 Microsampler, SciPro). Extrapolation from zero-flow analysis was performed by fitting single-phase exponential decay curve to the mean apoE concentrations at varying flow rates with the constraint that the plateau 
was set to 0 . Percent recovery for each flow rate was calculated using $C_{x} / E * 100$, where $C_{x}$ was the apoE concentration at a given flow rate and $E$ was the estimated apoE concentration at steady-state.

\section{In vivo microdialysis}

Mice were anesthetized using 1.5\%-2.5\% isoflurane, the head shaved, and an anterior to posterior incision made along the midline of the head to expose the skull from several $\mathrm{mm}$ anterior of bregma to several $\mathrm{mm}$ posterior of lambda. The mouse was then mounted onto a manipulator arm-equipped small animal stereotaxic apparatus (David Kopf Instruments, Tujunga, CA, USA). The skull was then leveled to within $0.1 \mathrm{~mm}$ at lambda, bregma, and two points $2.2 \mathrm{~mm}$ lateral of midline. A bore hole $(1.0 \mathrm{~mm}$ diameter $)$ was then created above the left hippocampus (bregma $-3.1 \mathrm{~mm}, 2.5 \mathrm{~mm}$ lateral, dura mater $-0.6 \mathrm{~mm})$. A second bore hole $(0.75 \mathrm{~mm})$ was placed in the right, anterior quadrant of the skull in which to place an anchoring bone screw. An AtmosLM Guide Cannula (PEG-X, Eicom, San Diego, CA, USA) was then stereotactically inserted into the left hippocampal formation $\left(12^{\circ}\right.$ angle, dura mater $\left.-1.2 \mathrm{~mm}\right)$. The cannula was then secured into place using a binary dental cement. An AtmosLM Dummy Cannula (PED-X, Eicom, San Diego, CA, USA) was then inserted into the guide cannula and secured with a plastic cap nut. The wound was then closed using surgical adhesive and the animal placed into a clean cage and provided with access to food and water ad libitum.

1,000 kDa microdialysis probes (AtmosLM Microdialysis probe, PEP-X-OY, Eicom, San Diego, CA, USA) were prepared as described above. Mice were then briefly anesthetized with isoflurane and probes were inserted into the hippocampus through the guide cannula and the mouse fitted with a plastic collar. Mice were then placed into a cage designed to allow for free movement without placing stress on the Teflon tubing or probe apparatus (Raturn Stand-Alone System, BASi, West Lafayette, IN, USA). The mice were kept under constant light conditions for the remainder of the experiment. Peristaltic pumps were operated at maximum speed for two hours to prevent clogging of the microdialysis membrane. Flow rates were then reduced to $1.0 \mu \mathrm{L} / \mathrm{min}$ to measure apoE. Samples were collected bi-hourly using a refrigerated fraction collector (Univentor 820 Microsampler, SciPro).

\section{ApoE and $A \beta$ ELISAs}

The apoE concentration in microdialysis samples were analyzed by an apoE sandwich ELISA. For human apoE, a 96-well plate (Nunc) was coated with $500 \mathrm{ng} /$ well $\mathrm{HJ}$ 6.2 overnight at $4^{\circ} \mathrm{C}$. For murine apoE, $1 \%$ milk served as the capture ligand. The plate was then blocked with $1 \%$ milk for $60 \mathrm{~min}$. at $37^{\circ} \mathrm{C}$. Microdialysis samples were diluted with our standard ELISA buffer (0.5\% BSA, 0.025\% Tween-20 in PBS, pH 7.4). For human apoE isoforms recombinant apoE3 standards were diluted with ELISA buffer and microdialysis perfusion buffer to match the buffer composition of microdialysis samples. For quantification of murine apoE, pooled C57BL/6J plasma was used as a standard. Samples and standards were loaded and incubated overnight at $4^{\circ} \mathrm{C}$. Biotinylated $\mathrm{mHJ} 6.3$ or $\mathrm{mHJ} 6.1$ was used as a detection antibody for mouse or human apoE, respectively. Following a $90 \mathrm{~min}$. incubation at $37^{\circ} \mathrm{C}$ with detection antibody, poly-streptavidin-horseradish peroxidase (HRP) (Thermo Scientific) was applied to the plate and incubated for $90 \mathrm{~min}$. at room temperature. ELISAs were then developed using Super Slow ELISA TMB (Sigma) and read using a Bio-Tek FL-600 plate reader at $650 \mathrm{~nm}$. A $\beta_{x-40}$ was analyzed by sandwich ELISA as described previously, using $\mathrm{HJ} 2$ as the capture antibody and biotinylated HJ 5.1 as the detection antibody [8].

\section{Nondenaturing gradient gel electrophoresis and western blotting}

Microdialysis, CSF, or microdialysis buffer samples were diluted with Native PAGE sample buffer (Life Technologies, Carlsbad, CA, USA) and electrophoresed on a 4-20\% Tris-Glycine gel (Life Technologies, Carlsbad, CA, USA) at $100 \mathrm{~V}$ for 24 hours at $4{ }^{\circ} \mathrm{C}$. A mixture of proteins with defined hydrated diameters was used for size standards (Amersham $^{\mathrm{Tm}}$ HMW Calibration Kit for Native Electrophoresis, Cat. \# 17-0445-01, GE Healthcare). Proteins were transferred to a nitrocellulose membrane and probed with antibodies recognizing goat anti-human apoE (50AG1b, 1:200, Academy Biomedical). Membranes were then washed extensively and probed with a donkey anti-goat IgG conjugated to HRP (1:5000, sc-2056, Santa Cruz Biotechnology). Blots were then developed using enhanced chemiluminescence (Lumigen-TMA6, Lumigen Inc.) and imaged with a Syngene G-Box (Syngene, Cambridge, UK).

\section{Statistical analysis}

Statistical analysis was performed using PRISM version 5.0 (GraphPad). Data are presented as mean \pm SEM. Comparisons between two groups were made using a two-tailed unpaired t-test. Multiple groups were compared using ANOVA followed by Tukey's post hoc test. Statistical significance was assigned to $\mathrm{p}$-values less than 0.05 .

\section{Competing interests}

$\mathrm{DMH}$ co-founded and is on the scientific advisory board of C2N Diagnostics and currently serves as a consultant for Astra Zeneca, Bristol Myers Squibb, and Genentech. GEL co-founded ReXceptor, Inc.

\section{Authors' contributions}

JDU, GEL, JRC, JMC and DMH conceived and designed the experiments. JDU, JMC, TEM and DRS performed microdialysis experiments. JDU, JMC and JMB analyzed microdialysis samples. JLR, JMB, and JRC performed bexarotene 
experiments. HJ prepared CSF samples. JDU and PBV performed native gel electrophoresis analysis. JDU wrote the paper and JRC and DMH revised the paper. All authors gave final approval of the version to be published. All authors read and approved the final manuscript.

\section{Acknowledgments}

This work was supported by R37 AG13956 (DMH), Ellison Medical Foundation Senior Scholar Award (DMH), the Cure Alzheimer's Fund (DMH), R01 AG042513 (JRC), P01 NS07496901 (JRC), K01 AG029254 (JRC). We thank Floy Stewart, Kaoru Yamada, and Mary Beth Finn for expert technical advice and assistance. ApoE2 Kl, apoE3 Kl, and apoE4 Kl mice were generously provided by Dr. Patrick M. Sullivan (Duke University).

\section{Author details}

'Department of Neurology, Saint Louis, MO, USA. ${ }^{2}$ Hope Center for Neurological Disorders, Saint Louis, MO, USA. ${ }^{3}$ Developmental Biology, Saint Louis, MO, USA. ${ }^{4}$ Knight Alzheimer's Disease Research Center, Washington University School of Medicine, Saint Louis, MO 63110, USA. ${ }^{5}$ Department of Neurosciences, Case Western Reserve University, School of Medicine, Cleveland, OH, 44106 USA.

Received: 29 January 2013 Accepted: 16 April 2013

Published: 19 April 2013

\section{References}

1. Vance JE, Hayashi H: Formation and function of apolipoprotein Econtaining lipoproteins in the nervous system. Biochimica Biophysica Acta (BBA) Molecular Cell Biol Lipids 2010, 1801:806-818.

2. Holtzman DM, Herz J, Bu G: Apolipoprotein e and apolipoprotein e receptors: normal biology and roles in Alzheimer disease. Cold Spring Harb Perspect Med 2012, 2:a006312.

3. Castellano JM, Kim J, Stewart FR, Jiang H, DeMattos RB, Patterson BW, Fagan AM, Morris JC, Mawuenyega KG, Cruchaga C, et al: Human apoE Isoforms Differentially Regulate Brain Amyloid- $\beta$ Peptide Clearance. Sci Trans/ Med 2011, 3:89ra57.

4. Hashimoto T, Serrano-Pozo A, Hori Y, Adams KW, Takeda S, Banerji AO, Mitani A, Joyner D, Thyssen DH, Bacskai BJ, et al: Apolipoprotein E, Especially Apolipoprotein E4, Increases the Oligomerization of Amyloid $\beta$ Peptide. J Neurosci 2012, 32:15181-15192.

5. Wahrle SE, Jiang H, Parsadanian M, Kim J, Li A, Knoten A, Jain S, HirschReinshagen $\mathrm{V}$, Wellington $\mathrm{CL}$, Bales KR, et al: Overexpression of $A B C A 1$ reduces amyloid deposition in the PDAPP mouse model of Alzheimer disease. J Clin Invest 2008, 118:671-682.

6. Hirsch-Reinshagen V, Zhou S, Burgess BL, Bernier L, Mclsaac SA, Chan JY, Tansley GH, Cohn JS, Hayden MR, Wellington CL: Deficiency of ABCA1 Impairs Apolipoprotein E Metabolism in Brain. J Biol Chem 2004 279:41197-41207.

7. Kim J, Jiang H, Park S, Eltorai AEM, Stewart FR, Yoon H, Basak JM, Finn MB, Holtzman DM: Haploinsufficiency of Human APOE Reduces Amyloid Deposition in a Mouse Model of Amyloid- $\beta$ Amyloidosis. J Neurosci 2011 , 31:18007-18012.

8. Cramer PE, Cirrito JR, Wesson DW, Lee CYD, Karlo JC, Zinn AE, Casali BT, Restivo JL, Goebel WD, James MJ, et al: ApoE-Directed Therapeutics Rapidly Clear $\beta$-Amyloid and Reverse Deficits in AD Mouse Models. Science 2012, 335:1503-1506.

9. Kim J, Eltorai AEM, Jiang H, Liao F, Verghese PB, Kim J, Stewart FR, Basak JM, Holtzman DM: Anti-apoE immunotherapy inhibits amyloid accumulation in a transgenic mouse model of $A \beta$ amyloidosis. J Exp Med 2012, 209:2149-2156.

10. Chefer VI, Thompson AC, Zapata A, Shippenberg TS: Overview of Brain Microdialysis. Curr Protoc Neurosci 2009, 47:7.1.1-7.1.28

11. Cirrito JR, Yamada KA, Finn MB, Sloviter RS, Bales KR, May PC, Schoepp DD, Paul SM, Mennerick S, Holtzman DM: Synaptic Activity Regulates Interstitial Fluid Amyloid- $\beta$ Levels In Vivo. Neuron 2005, 48:913-922

12. Bero AW, Yan P, Roh JH, Cirrito JR, Stewart FR, Raichle ME, Lee J-M, Holtzman DM: Neuronal activity regulates the regional vulnerability to amyloid-[beta] deposition. Nat Neurosci 2011, 14:750-756.

13. Roh JH, Huang Y, Bero AW, Kasten T, Stewart FR, Bateman RJ, Holtzman DM: Disruption of the Sleep-Wake Cycle and Diurnal Fluctuation of $\beta$ Amyloid in Mice with Alzheimer's Disease Pathology. Sci Transl Med 2012, 4:150ra122.
14. Cirrito JR, May PC, O'Dell MA, Taylor JW, Parsadanian M, Cramer JW, Audia JE, Nissen JS, Bales KR, Paul SM, et al: In Vivo Assessment of Brain Interstitial Fluid with Microdialysis Reveals Plaque-Associated Changes in Amyloid- $\beta$ Metabolism and Half-Life. J Neurosci 2003, 23:8844-8853.

15. Takeda S, Sato N, Ikimura K, Nishino H, Rakugi H, Morishita R: Novel microdialysis method to assess neuropeptides and large molecules in free-moving mouse. Neuroscience 2011, 186:110-119.

16. Verges DK, Restivo JL, Goebel WD, Holtzman DM, Cirrito JR: Opposing Synaptic Regulation of Amyloid- $\beta$ Metabolism by NMDA Receptors In Vivo. J Neurosci 2011, 31:11328-11337.

17. Mandrekar-Colucci S, Karlo JC, Landreth GE: Mechanisms Underlying the Rapid Peroxisome Proliferator-Activated Receptor- $\gamma$-Mediated Amyloid Clearance and Reversal of Cognitive Deficits in a Murine Model of Alzheimer's Disease. J Neurosci 2012, 32:10117-10128.

18. Fan J, Stukas S, Wong C, Chan J, May S, DeValle N, Hirsch-Reinshagen V, Wilkinson A, Oda MN, Wellington CL: An ABCA1-independent pathway for recycling a poorly lipidated $8.1 \mathrm{~nm}$ apolipoprotein E particle from glia. J Lipid Res 2011, 52:1605-1616.

19. DeMattos RB, Brendza RP, Heuser JE, Kierson M, Cirrito JR, Fryer J, Sullivan PM, Fagan AM, Han X, Holtzman DM: Purification and characterization of astrocyte-secreted apolipoprotein $\mathrm{E}$ and J-containing lipoproteins from wild-type and human apoE transgenic mice. Neurochem Int 2001, 39:415-425

20. Wahrle SE, Jiang H, Parsadanian M, Hartman RE, Bales KR, Paul SM, Holtzman DM: Deletion of Abca1 Increases A $\beta$ Deposition in the PDAPP Transgenic Mouse Model of Alzheimer Disease. J Biol Chem 2005, 280:43236-43242.

21. Fagan AM, Holtzman DM, Munson G, Mathur T, Schneider D, Chang LK, Getz GS, Reardon CA, Lukens J, Shah JA, LaDu MJ: Unique Lipoproteins Secreted by Primary Astrocytes From Wild Type, apoE (-l-), and Human apoE Transgenic Mice. J Biol Chem 1999, 274:30001-30007.

22. Bales KR, Liu F, Wu S, Lin S, Koger D, DeLong C, Hansen JC, Sullivan PM, Paul SM: Human APOE Isoform-Dependent Effects on Brain $\beta$-Amyloid Levels in PDAPP Transgenic Mice. J Neurosci 2009, 29:6771-6779.

23. Riddell $D R$, Zhou $H$, Atchison $K$, Warwick HK, Atkinson PJ, Jefferson J, Xu L, Aschmies S, Kirksey Y, Hu Y, et al: Impact of Apolipoprotein E (ApoE) Polymorphism on Brain ApoE Levels. J Neurosci 2008, 28:11445-11453.

24. Liu CC, Kanekiyo T, Xu H, Bu G: Apolipoprotein E and Alzheimer disease: risk, mechanisms and therapy. Nat Rev Neurol 2013, 9:106-118.

25. Rosenbaum Al, Maxfield FR: Niemann-Pick type $\mathrm{C}$ disease: molecular mechanisms and potential therapeutic approaches. J Neurochem 2011, 116:789-795.

26. Sullivan PM, Mace BE, Maeda N, Schmechel DE: Marked regional differences of brain human apolipoprotein e expression in targeted replacement mice. Neuroscience 2004, 124:725-733.

27. Fagan AM, Younkin LH, Morris JC, Fryer JD, Cole TG, Younkin SG, Holtzman DM: Differences in the Abeta40/Abeta42 ratio associated with cerebrospinal fluid lipoproteins as a function of apolipoprotein $\mathrm{E}$ genotype. Ann Neurol 2000, 48:201-210.

doi:10.1186/1750-1326-8-13

Cite this article as: Ulrich et al:: In vivo measurement of apolipoprotein $\mathrm{E}$ from the brain interstitial fluid using microdialysis. Molecular Neurodegeneration 2013 8:13.

\section{Submit your next manuscript to BioMed Central and take full advantage of:}

- Convenient online submission

- Thorough peer review

- No space constraints or color figure charges

- Immediate publication on acceptance

- Inclusion in PubMed, CAS, Scopus and Google Scholar

- Research which is freely available for redistribution 